\title{
REVIEW \\ Study on Strengthening Supervision of the Third-party Laboratory by Suzhou Municipal Government
}

\author{
Chaoyong Liu* Yucai Wu \\ Jiangsu University of Science and Technology, Zhenjiang, Jiangsu, 212003, China
}

\section{ARTICLE INFO}

Article history

Received: 25 March 2021

Accepted: 9 April 2021

Published Online: 30 June 2021

Keywords:

Government

Third-party laboratory

Supervision

\begin{abstract}
As public service testing structure of testing products, the quality of product testing report issued by the third-party laboratory has a great impact on the healthy development of market economy. In the face of the new situation of rapid economic development, the third-party laboratory develops rapidly, but the government supervision is not in place, which restricts the healthy and orderly development of the third-party laboratory. Therefore, it is very necessary to study how the government effectively supervises the thirdparty laboratory. This paper takes Suzhou as an example. On the basis of in-depth investigation, using the theory of service government, this paper analyzes the current situation of Suzhou municipal government's supervision of third-party laboratories, and probes into the problems existing in the supervision and management system, the supervision is not comprehensive, and the independence of the supervision team is not strong. Based on the advanced experience of foreign countries in supervising the third-party laboratory, this paper puts forward some countermeasures and suggestions to promote the Suzhou municipal government to strengthen the supervision of the third-party laboratory from the aspects of improving the implementation rules of laws and regulations, increasing the investment, strengthening the equipment update and team building, increasing the punishment for violations of laws and regulations, and giving full play to the masses and industry supervision.
\end{abstract}

\section{Introduction}

With the rapid development of China's economy and society, people's living standards are increasing, and people put forward higher requirements for product quality. The third-party laboratory, independent of the impartial and authoritative commodity testing institution, emerged. This is conducive to improving the level of product detection, improving the quality of goods, providing a strong service platform and support for the transformation and upgrading of industry, and helping the government get rid of the "Trust
Crisis". In order to standardize the development of the third-party laboratory, the Metrology Law of the People's Republic of China was adopted at the 12th meeting of the Standing Committee of the Sixth National People's Congress on September 6th, 1985, but it has been developing slowly. Until the end of 1990s, the inspection and testing industry was officially open to the market, and the third-party laboratory grew rapidly. By the end of 2018, 9365 thirdparty laboratories approved by CNAS have been approved in China, and the operating income of the inspection and testing industry has reached 28.05 billion yuan. Over the

*Corresponding Author:

Chaoyong Liu,

Jiangsu University of Science and Technology, Zhenjiang, Jiangsu, 212003, China;

Email:18120091119@163.com 
past 20 years, the third-party laboratory has experienced the development from small to large, from weak to strong. The quantity and turnover are rising rapidly at a rate of more than $15 \%$ per year. It is expected that the next five years will still develop at a compound growth rate of $16.24 \%$.

It should be noted that due to the complex structure of the main body of the third-party laboratory, involving a wide range of industries, high technical requirements, its rapid development at the same time, there are uneven quality of the team, different quality of testing equipment, different levels of testing standards, and even a small number of units issued false reports. It is urgent for the government to strengthen supervision, promote the standardized service of the thirdparty laboratory, and improve the health of the third-party laboratory orderly development. At present, there are still many problems in the government supervision, such as the multi supervision subject, the ineffective implementation of supervision policies, the weak supervision force, and the inadequate supervision work, resulting in many problems in the supervision process of the third-party laboratory. In order to strengthen the effective supervision, the state has issued a series of standards and regulations, which provide the basis for the government and relevant public organizations to supervise the third-party laboratory. Therefore, the relevant government departments and public organizations need to strengthen the research on how to effectively enforce the law, strengthen the supervision of the third-party laboratory and improve the service quality ${ }^{[1]}$.

Suzhou is located in the forefront of reform and opening up, and its economy is very developed. By the end of 2018, there were 609 inspection and testing institutions in Suzhou, with 689 CMA and CNAS certificates. Relatively speaking, the types of thirdparty laboratories are relatively complete. Based on the analysis of the current situation and existing problems of Suzhou municipal government's supervision of thirdparty laboratories by using public product supply theory, collaborative governance theory and other theories, this paper proposes to promote the effective supervision of third-party laboratories by Suzhou municipal government and improve the quality of third-party laboratories The countermeasures and suggestions of its service also provide reference for other regions ${ }^{[2]}$.

\section{Analysis on the Current Situation of the Third-party Laboratory Supervised by Suzhou Municipal Government}

\subsection{Development Status of the Third-party Laboratory in Suzhou}

By the end of 2018, there were 609 third-party laboratories in Suzhou, with 689 CMA and CNAS certificates. The total value of fixed assets of laboratory equipment and supporting devices was 8.469 billion yuan, the laboratory area was 1.0242 million square meters, 19983 employees, and 6.2418 million inspection and testing reports were issued. Among them, there are 81 inspection and testing institutions above Designated Size (with inspection and testing income of 10 million yuan or more or with more than 50 employees at the end of the year), accounting for $21.09 \%$ of the total number of third-party laboratories, and accounting for $77.62 \%$ of the total income of the institutions. Among them, there are 12 laboratories with a total income of more than 100 million yuan. Among the third-party laboratories, there are 214 small institutions with a total income of less than 5 million yuan, accounting for $55.73 \%$ of the total number of institutions, but the income only accounts for $4.86 \%$. The total service revenue was about 6.154 billion yuan, an increase of $19.13 \%$ over 2017; it accounted for $2.19 \%$ of the total revenue of the third-party laboratories in China, accounting for $0.33 \%$ of Suzhou's GDP in 2018; the total profit was about 1.367 billion yuan, with an average profit margin of $22.21 \%$. The service sector covers almost all sectors of Suzhou's national.

As a third-party laboratory in high-tech service industry, relevant research shows that there is a high correlation between its service income and equipment input. The equipment configuration of the third-party laboratory in Suzhou has proved this in recent three years. At the end of 2018, the original value of service equipment of 12 third-party laboratories with income of more than RMB 100 million is very high, and the equipment is advanced and complete in types. The total original value of equipment accounts for $47.71 \%$ of the total equipment value of the third party laboratory in Suzhou. The service income accounts for $42.22 \%$ of the total industry revenue. At the same time, the third-party laboratory industry is also a talented and technology intensive industry. Currently, 19983 employees engaged in the third-party laboratory in Suzhou are sorted out, including $56.51 \%$ of the undergraduate and above, and $26.97 \%$ of the middle and senior technical titles. In recent three years, the number of employees in the third-party laboratory industry has increased by the largest in 2017, reaching $35.83 \%$, which has declined in 2018, mainly due to the fact that 13 qualification agencies and 29 national accreditation (CNAS) laboratories have not submitted survey statistics this year ${ }^{[3]}$.

\subsection{Analysis of the Current Situation of the Third-party Laboratory Supervised by Suzhou Municipal Government}

In view of the possible problems of the third-party 
laboratory service, Suzhou municipal government has established a perfect supervision organization for the third party laboratory with clear division of labor. The municipal level is undertaken by Suzhou market supervision and Administration Bureau and Suzhou quality inspection and Certification Association. The supervision institutions of county-level cities and districts are managed and the third-party laboratories are monitored irregularly. Especially, serious problems arise the laboratory focuses on inspection, and the supervision agencies of county-level cities and districts are mainly responsible for the supervision of the daily work of the third-party laboratories.

In order to strengthen the supervision of the thirdparty laboratory, the state has issued a series of laws and regulations. As early as September 6, 1985, the 12th meeting of the Standing Committee of the Sixth National People's Congress passed the Metrology Law of the People's Republic of China, which was amended on October 26, 2018. On October 16, 2017, China National Certification and Accreditation Administration issued the certification and accreditation industry standard $\mathrm{Rb} / \mathrm{T}$ of the people's Republic of China On March 1, 2018, CNAS-CL01 accreditation criteria for testing and calibration laboratories (ISO /IEC 17025:2017) was issued by China National Accreditation Committee for conformity assessment; on March 20, 2019, Wang Yupu, Minister of the Ministry of emergency management of the people's Republic of China, issued the No.1 order of the Ministry of emergency management of the people's Republic of China management measures for safety evaluation, testing and inspection institutions. Suzhou municipal government and relevant public organizations conscientiously implement the national laws and regulations on the supervision of third-party laboratories, and strictly deal with illegal acts. At the same time, provide effective services for the third party laboratory. For example, the third-party laboratory should provide guidance for the application for qualification, encourage the voluntary application for the accreditation of the third-party laboratory and the accreditation of the laboratory qualification, the China conformity assessment and Accreditation Commission and the provincial market supervision and Administration Commission should entrust experts to carry out document audit and on-site audit, and issue CNAS and CMA qualification certificates. If problems are found in the supervision, the municipal and county level market supervision and Administration Bureau shall timely report to China conformity assessment and Accreditation Commission and provincial level market supervision and Administration Bureau. China conformity assessment and Accreditation Commission and provincial market supervision and Administration Bureau follow up the seriousness of the problem, and give warning, suspension of qualification, cancellation of qualification and other penalties. The third party laboratory without CNAS and CMA qualification shall not carry out relevant experiments or issue test reports with certification ${ }^{[4]}$.

Under the premise that the relevant departments and public organizations of the state conscientiously implement the national laws and regulations, the thirdparty Laboratory of Suzhou city shall be supervised and reviewed by the China Certification and Accreditation Commission and the provincial market supervision administration every 1-2 years, and participate in the ability verification organized by authoritative institutions and the supervision institutions of county-level cities and districts in the irregular "flight inspection" and regular requirements of the Suzhou market supervision administration Under the daily management, once problems are found, the third-party laboratory should be dealt with immediately to ensure the continuous and effective detection ability and quality control of the thirdparty laboratory, which also promotes the third-party laboratory to regularly test equipment for measurement calibration in time, and submit the measurement calibration certificate to China Certification and Accreditation Commission, provincial market supervision administration or Suzhou market supervision bureau Supervise the authority for inspection at any time. Of course, if a third-party laboratory encounters complaints or reports, Suzhou market supervision bureau will conduct a special audit on the third-party laboratory. Through a series of supervision, in recent years, Suzhou thirdparty laboratory has not had any major quality accidents. In recent years, the number and turnover of third-party laboratories in Suzhou have maintained a steady growth trend, the detection level has been continuously improved, and the competitiveness in the country has also been continuously enhanced ${ }^{[5]}$.

\section{Analysis of the Main Problems and Causes of Suzhou Municipal Government's Supervision of the Third-party Laboratory}

\subsection{Main Problems}

(1) The Supervision and Management System Needs to Be Further Straightened Out

Although Suzhou municipal government stipulates that Suzhou and the market supervision and administration of each county (District) and Suzhou quality inspection and Certification Association are the third party laboratory 
institutions, in the actual work process, different levels of government institutions supervise the third party laboratories, such as Jiangsu provincial market supervision and administration and Suzhou market supervision and Administration Bureau both supervise the third party laboratories in Suzhou The Fang laboratory shall supervise. At the same time, due to the wide range of industries involved in the third-party laboratory, cross supervision will occur among different government departments. For example, Suzhou Environmental Protection Bureau and Safety Supervision Bureau often focus on the third-party laboratory of environmental testing, and the supervision content of the same third-party laboratory is basically the same, but they are carried out separately. To some extent, the repeated supervision has affected the normal operation of the third-party laboratory, increased its burden and affected the efficiency of the government. This requires the use of relevant supporting rules.

(2) Not Effective to Achieve Comprehensive Supervision

From the survey, Suzhou City has not yet achieved the supervision of the third-party laboratory. The supervision and law enforcement of some unqualified third-party laboratories are not effective. According to the laws and regulations, the unqualified illegal laboratory is unable to carry out the testing work and issue the testing report. But at present, according to incomplete statistics, there are more than 100 laboratories in Suzhou, or only registered in industry and commerce, without applying for any qualification, the China conformity assessment and Accreditation Commission and the provincial market supervision bureau can not supervise them according to the requirements of CNAS and CMA, that is, they do not have the testing qualification; or the third-party laboratory whose qualification has been cancelled can not be called the third-party laboratory in a strict sense They're working in a third-party lab. These two types are not qualified for testing and should be banned according to law, but they are still engaged in testing work. What's more serious is the issue of very poor quality reports, or some even directly in accordance with the requirements of customers to issue false reports, which seriously interferes with the impartiality and reputation of the third-party laboratory, but at present there is no supervision. At the same time, the current part of the qualified private and foreign thirdparty laboratory supervision. Most of the private or foreign-funded third-party laboratories have CNAS and CMA qualifications. In the promotion, CNAS and CMA qualifications are vigorously promoted to improve their social reputation and attract customers. However, in practice, they do not fully comply with the requirements of CNAS and CMA. In order to evade supervision, they all stamp CNAS and CMA in their reports. Because they are not stamped with CNAS and CMA, there is no way to supervise these reports. According to the survey, about $70 \%$ of the private or foreign-funded third-party laboratories have this kind of illegal phenomenon. In the long run, it will damage the market and reputation of the third-party laboratories in the city. Therefore, it is necessary to strengthen the supervision and punishment.

(3) The Independence of Supervision Team is Not Strong

At present, the supervision team of the third-party laboratory in Suzhou is basically composed of experts drawn from the state-owned or collective owned thirdparty laboratories, although the experts of the transferred units can not participate in the inspection of their own units and implement the avoidance system. However, they are mainly transferred from large testing institutions to get to know each other. In this way, when the government departments or public organizations supervise the thirdparty laboratories, the state-owned or collective thirdparty experiments are not only the subject of supervision, but also the object of supervision, and the objectivity and impartiality of supervision are not high. At the same time, it should be noted that the experts of the state-owned or collective third-party laboratories who are transferred to supervise the personnel of other third-party laboratories on behalf of the government are basically the internal audit experts of their own units. They supervise both other third-party laboratories and internal laboratories. As a result, these experts are not only "referees", but also "athletes". It is easy for them to have "black under the light" self-regulation, which leads to the lack of supervision. Therefore, it is very necessary to establish an independent supervision team.

\subsection{Causes Analysis}

(1) The Supervision System is Not Perfect

A sound and perfect supervision system is the basis and premise for the government to effectively supervise the third-party laboratory. Especially for the lack of supervision on the state-owned or collective owned third-party laboratories, and the lack of supervision on the laboratories that have not applied for qualification, or the laboratories that have applied for qualification but have not been approved or whose qualification has been revoked and are still engaged in testing work, we should improve the relevant supervision system, establish and improve the supervision system, and form a comprehensive supervision basis. Of course, due to the gap between the government's management performance 
and the government's requirements for the supervision of third-party laboratories, under the current situation, the relevant government departments generally implement the supervision according to the principle of "no authorization can not be done without law". They prefer not to do or less, rather than take the responsibility and risk to themselves. As a result, the supervision has no strong motivation to actively strengthen the supervision of thirdparty laboratories. He is in charge of the work. We need to improve the system.

(2) Professional Testing Ability Needs to be Improved

With the rapid development of high-quality economy, the detection of new products is increasing, which requires the government to speed up the update of professional testing equipment. The introduction of new technical personnel, enrich the testing force and improves the detection level. However, at present, Suzhou municipal government is faced with a large amount of work, heavy task, great difficulty and strong professionalism, especially in the regulatory field with high technical requirements. It is difficult for the government to invest enough manpower and financial resources to update professional equipment and introduce high-level professionals. At the same time, the mature supervision experience of some professional testing talents often have the heart of supervision, but not the power of supervision. It can be seen that due to the lack of funds, the comprehensive supervision and detection ability is obviously insufficient.

(3) The Way of Supervision is Backward

At present, the supervision of the third-party laboratory by Suzhou municipal government is still based on the traditional supervision mode, which can not meet the needs of the market development to speed up the supervision of the third-party laboratory. In most cases, the government supervision is carried out by a single department, lacking of coordination and communication between departments, resulting in repeated supervision. In terms of supervision, there are overlapping phenomena. If it's good for us, we should emphasize the power of departments, which leads to excessive supervision; if it's bad for us, we should pass the buck to each other, so that the supervision of the third-party laboratory can't be solved for a long time. For example, in terms of market access, daily supervision and other work, there is a "two skin" phenomenon. Some local governments only examine and approve without supervision, while other departments turn a blind eye to the existing risks because they are not examined and approved by them. As a result, these institutions carry out all kinds of illegal activities under the "legal coat", brewing all kinds of potential risks.

\subsection{Experience of Foreign Testing for Third-party Laboratory}

The U.S. government has always advocated market freedom and is not easy to interfere in market behavior. The third-party laboratory in the United States is completely open to the market, and any competent laboratory can carry out the related work of the third-party laboratory. At present, according to the theory of public goods and the theory of government behavior, some scholars require the government to closely supervise the pharmaceutical and food industries. Even if necessary, it will be taken over and operated by the government.

The attitude of the Japanese government towards the third party laboratory has gone through the process of loosening, strictness and re easing. The number and scale of the third-party laboratories in Japan are relatively small, and most of the third-party laboratories only participate in the Japanese domestic market, do not participate in the world market competition, and have relatively little contact with international laboratories. At present, the supervision of the third-party laboratory by the Japanese government is mainly completed by industry associations, and the supervision efficiency is relatively high.

\section{Countermeasures and Suggestions for Suzhou Municipal Government to Strengthen the Supervision of the Third-party Laboratory}

\subsection{Improve the Implementation Rules of Laws and Regulations for the Government to Supervise the Third-party Laboratory}

In view of the current situation of ineffective supervision of third-party laboratories, Suzhou municipal government should, in accordance with the national laws and regulations, combine with the actual situation of Suzhou City, from the aspects of supervision system, fund investment, supervision equipment update, supervision talent team construction, supervision procedure, punishment for illegal detection and false report, rewards and punishments for supervision in place and absence, and treatment of illegal rules To formulate relatively perfect supervision implementation rules, and seriously implement the implementation rules, so that the government's supervision of the third-party laboratory can be truly implemented, and constantly improve the quality of supervision.

\subsection{Establish a Unified Regulatory Body}

The establishment of a coordinated and unified government supervision system with clear rights 
and responsibilities is an important guarantee for the realization of comprehensive supervision of third-party laboratories. Therefore, according to the current situation that Suzhou Environmental Protection Bureau and Safety Supervision Bureau also participate in the supervision of the third-party Laboratory of environmental testing, the municipal government should take the lead, integrate the human and material resources of multiple departments, clearly divide the powers and responsibilities of various departments, and coordinate to form a unified supervision body led by the market supervision and Administration Bureau and coordinated by other departments, so as to avoid the occurrence of environmental pollution At present, the phenomenon of multi supervision is to realize comprehensive supervision, improve the efficiency of government supervision on the third party laboratory, and reduce the phenomenon of repeated supervision.

\subsection{Increase the Investment in the Supervision of the Third-party Laboratory}

According to the theory of service-oriented government, as the government of the people, providing public goods and services is the basic function of the government. The third-party laboratory supervision is to ensure the quality of testing products, let the people use health and safety products and services, and promote the healthy and orderly development of social economy. It is the basic responsibility of the government to serve the people. In view of the current situation of obsolete testing equipment, weak independence of the team and insufficient ability of the testing personnel in the third-party laboratory supervision in Suzhou, we should increase the investment in supervision, update the testing equipment, increase the training of the testing personnel, establish an independent testing team, improve the ability and level of the thirdparty laboratory supervision, realize all-round supervision, and ensure the quality of production To avoid the phenomenon of "fake lamp".

\subsection{Increase the Punishment for Violation of Laws and Regulations}

It is the premise to strengthen the crackdown on illegal behaviors to ensure the healthy development of the thirdparty laboratory. Therefore, the market supervision and administration of Suzhou Municipal Government shall ban those who do not have the qualification for testing, but are still engaged in the inspection work, and shall be banned according to law and fined. For the report with very poor quality, or false report according to the requirements of customers, or some private or foreign third-party laboratories have CNAS and CMA qualifications, they should publicize vigorously during publicity and promotion. However, in order to avoid supervision and regulations, the violation without CNAS and CMA chapters is not covered, supervision should be strengthened, punishment should be strengthened to make the testing market issue real product quality report The report will improve the credibility and social reputation and promote the healthy and orderly development of the third-party laboratory testing work.

\section{References}

[1] Chung R H, Kim B S, Abreu J M. Asian American multidimensional acculturation scale: development, factor analysis, reliability, and validity.[J]. Cultur Divers Ethnic Minor Psychol, 2004, 10(1):66-80.

[2] Hosmane B S. Improved likelihood ratio tests and pearson chi-square tests for independence in two dimensional contingency tables[J]. Communications in Statistics, 1986, 15(6):1875-1888.

[3] MatthewGoldstein, EdwardWolf, WilliamDillon. On a test of independence for contingency tables $[\mathrm{J}]$. Communications in Statistics, 1976, 5(2):159-169.

[4] Vincent and Elinor Ostrom. Public Goods and Public Choices, in Alternatives for delivering Public Services[M].E.S Savas, 1977: p7-14.

[5] Shinoi Sakata. Implementation of the OECD GLP principles at test facilities in Japan. Ann Ist Super Sanita 2018,44(4): 374-378. 